\title{
The Stor-e-Motion Visualization for Topic Evolution Tracking in Text Data Streams
}

\author{
Andreas Weiler, Michael Grossniklaus, and Marc H. Scholl \\ Department of Computer and Information Science, University of Konstanz \\ P.O. Box 188, 78457 Konstanz, Germany \\ firstname.lastname@uni-konstanz.de
}

Keywords: $\quad$ story visualization, text data streams, twitter

\begin{abstract}
Nowadays, there are plenty of sources generating massive amounts of text data streams in a continuous way. For example, the increasing popularity and the active use of social networks result in voluminous and fastflowing text data streams containing a large amount of user-generated data about almost any topic around the world. However, the observation and tracking of the ongoing evolution of topics in these unevenly distributed text data streams is a challenging task for analysts, news reporters, or other users. This paper presents "Store-Motion" a shape-based visualization to track the ongoing evolution of topics' frequency (i.e., importance), sentiment (i.e., emotion), and context (i.e., story) in user-defined topic channels over continuous flowing text data streams. The visualization supports the user in keeping the overview over vast amounts of streaming data and guides the perception of the user to unexpected and interesting points or periods in the text data stream. In this work, we mainly focus on the visualization of text streams from the social microblogging service Twitter, for which we present a series of case studies (e.g., the observation of cities, movies, or natural disasters) applied on real-world data streams collected from the public timeline. However, to further evaluate our visualization, we also present a baseline case study applied on the text stream of a fantasy book series.
\end{abstract}

\section{INTRODUCTION}

In recent years, there has been a continuous increase of social media services on the web. Unprecedented success and active usage of these services result in massive amounts of user-generated data. The amount of information in the generated data increases as well. For example, a large proportion of usergenerated content is automatically enriched by the geographical location of the user's device. As social media services changed the way we use the Internet and play an increasing role in our daily life, it was only a question of time until social media became a source for information gathering. Unfortunately, the vast amount and the high variability in the quality of user-generated data is obstructive to analysis tasks. But, at the same time, user-generated data enables us to extract interesting insights into a variety of different topics.

A popular example is the microblogging service Twitter. Initially introduced in 2006 as a simple platform for exchanging short messages ("tweets") on the Internet, Twitter rapidly gained worldwide popularity and has evolved into an extremely influential channel for broadcasting news and the means of real-time information exchange. Apart from its attractiveness as a means of communication-with over 140 million active users as of 2012 generating over 340 millions of tweets daily-Twitter has revolutionized the ways of exchanging information on the Internet and opened new ways for knowledge acquisition from social interaction streamed in real-time.

Due to the diversity of the provided information, Twitter even plays an increasingly important role as a source for news agencies. In fact, news agencies use Twitter for two important functionalities in their daily work. On the one hand, agencies use Twitter as a publication and distribution platform for current news articles with a high throughput rate. For example, any reproduction of a tweet ("retweet") reaches an average of about 1,000 users (Kwak et al., 2010). On the other hand, news agencies, such as BBC, are constantly increasing the usage of Twitter as a reference in their daily news reports (Tonkin et al., 2012). A further characteristic of Twitter is its vibrant user community with a wide range of different personalities from all over the world. The whole spectrum of use cases can be subdivided into a few categories of 
Twitter usage patterns, such as daily chatter, information and URL sharing, or news reporting (Java et al., 2007). Further research undertaken has discovered that the majority of users publish messages focusing on their personal concerns and matters, whereas a smaller set of users publish for information sharing (Naaman et al., 2010).

In this paper, we present an application for visually tracing and monitoring the importance, emotion, and story of user-defined topic channels in the continuous data stream of Twitter. Our work presents a compact visualization for time series event data, which supports users in identifying interesting data points in the large volume of tweets. Additionally, it is possible to overview whole sets of topics and to compare the evolution of different topics with each other over time. Furthermore, the application automatically displays the most influencing episode terms in a tag list over time. The observation of the evolution of importance, emotion, and story of topics is a task that needs to be done in various fields of analytics. For example, an analyst who wants to keep track of natural disasters appearing in the Twitter stream or any other news stream needs an appropriate application, which displays a compact overview of all appearances of the topic in the data. In the case studies section, we mainly focus on the visualization of text data streams of the social microblogging service Twitter. However, to further evaluate our visualization, we also present a case study applied on the text data stream of the fantasy book series "Harry Potter".

The remainder of this paper is structured as follows. We begin in Section 2 with a presentation of the state of the art and the background in which our work is situated. We then present the system design, including the implementation of the processing pipeline with Niagarino, and the design goals of the visualization in Section 3. In Section 4, we discuss a series of case studies that give qualitative evidence as to the validity of our approach. Finally, concluding remarks are given in Section 5.

\section{BACKGROUND}

A lot of research is being done on the analysis and knowledge discovery from social media data. As a good overview, Bontcheva et al. (Bontcheva and Rout, 2012) present a survey of sense making of social media data, which lists state-of-the-art approaches for mining semantics from social media streams. Because of the fast propagation speed of information in social media networks, a high number of research works focus on event or topic detec- tion and tracking for various domains. For example, Sakaki et al. (Sakaki et al., 2010) presented a system for earthquake detection and Weng et al. (Weng et al., 2011) a system to detect events during elections in the Twitter data stream. In addition to domain specific systems, open domain event detection systems, like "TwitInfo" (Marcus et al., 2011), "enBloque" (Alvanaki et al., 2012), and "TwiCal" (Ritter et al., 2012) tackle the challenge to detect events of all different kinds and present the results with various visualizations.

Further research is undertaken in the area of epidemics tracking (Culotta, 2010), situational awareness (MacEachren et al., 2011), and disaster management (Lee et al., 2012). However, none of these systems combine the dimensions importance, emotions, and story to visually guide the perception of the user to unexpected and interesting points or periods in time. Nevertheless, there are a number of works that emerged in the area of visual analytics for Twitter streams. For example, "SensePlace2" (MacEachren et al., 2011), supports overview and detail maps of tweets, place-time-attribute filtering of tweets, and analysis of changing issues and perspectives over time and across space as reflected in tweets. However, in contrast to our work, they use a crawler to systematically query the Twitter API for tweets containing any topics deemed to be of interest, instead of using the data stream directly.

"ScatterBlogs2" (Bosch et al., 2013) is another approach that lets analysts build task-tailored message filters in an interactive and visual manner based on recorded messages of well-understood previous events. In contrast to our work, it is possible to redefine filters and also to create more powerful filters. However, they do not provide an overview visualization to follow the evolution of topics over time and also do not include any information about emotions. Another work is presented by (Dork et al., 2010), which they called a visual backchannel for large scale events. They present a novel way of following and exploring online conversations about large-scale events using interactive visualizations in a timeline fashion.

Furthermore a series of research was done in the area of news messages visualization. Havre et al. described "ThemeRiver" (Havre et al., 2002), an approach, which uses a stacked graph to help users to identify time-related patterns, trends, and relationships across a large collection of documents. Most similar to our approach is the work proposed by Krstajić et al. (Krstajić et al., 2011), which presents a technique called "CloudLines" showing both the current and the historic amount of news for pre-defined topics and try to capture the problem of high density 
and over-plotting via an importance function. Another application in the area of news streams can be found in (Wanner et al., 2009). Further work is done with location reports (Overby et al., 2009) and forum posts (Wanner et al., 2011). In contrast to our proposed idea, which uses a fast, uneven, and noisy stream of short messages, almost all of the systems mentioned above are applied to well-structured text and lack the flexibility to add new topics on-the-fly and monitor topics from a continuous stream of data.

\section{SYSTEM DESIGN}

The main contribution of our approach is a visualization for tracking the evolution of self-defined topics by analysts, information seekers, or default users in the massive stream of Twitter data. The high volume and propagation rate of tweets makes it difficult for users to follow the evolution of topics inside the continuous data flow. Furthermore, it is a big challenge to discriminate between normal behavior of the topic evolution or unusual and abnormal behavior, which usually is an indicator for an interesting event in the context of a topic. Therefore, the visualization is tailored to support the characteristic of fast distribution and spreading of information of social media services. However, it can also be applied to other types of textual data like a book series (see Section 4.2).

In the following, we introduce the design of the Stor-e-Motion visualization and motivate the three major design goals of visualizing the evolution of the Importance, Emotion, and Story around a topic. Furthermore, we describe the different options for defining Topic Channels to follow individual defined topics in the data stream.

\subsection{PROCESSING PIPELINE}

In order to support the exchange and extension of components in the processing pipeline, we use Niagarino $^{1}$, a data stream management system that is developed and maintained by our research group. The main purpose of Niagarino is to serve as an easy-touse and extensible research platform for streaming applications such as the one presented in this paper. The concepts embodied by Niagarino can be traced back to a series of pioneering data stream management systems, such as Aurora (Abadi et al., 2003), Borealis (Abadi et al., 2005), and STREAM/CQL (Arasu et al., 2006). In particular, Niagarino is an offshoot of

\footnotetext{
${ }^{1}$ http: //www. informatik. uni-konstanz.de/ grossniklaus/software/niagarino/
}

NiagaraST (Li et al., 2008), with which is shares the most common ground. In this section, we briefly summarize the parts of Niagarino that are relevant for this paper. Niagarino is implemented in Java 8 and relies heavily on its new language features. In particular, anonymous functions ( $\lambda$-expressions) are used in several operators in order to support lightweight extensibility with user-defined functionality. The current implementation runs every operator in its own thread. Operator threads are scheduled implicitly using fixedsize input/output buffers and explicitly through backwards messages.

In Niagarino, a query is represented as a directed acyclic graph $Q=(O, S)$, where $O$ is the set of operators used in the query and $S$ is the set of streams used to connect the operators. The query plan of a single topic channel in the Stor-e-Motion visualization is shown in Figure 1. Each query plan emits the results to the visualization node, which continuously updates and visualizes the results. Niagarino implements a series of operators. The selection $(\sigma)$ and projection $(\pi)$ operator work exactly the same as their counterparts in relational databases. In our case, we use them to select tuples corresponding to the topic channel definition. For the selection operator, we use different predicates (keyword selection and geographical based selection) to express the channel definition.

As shown in Figure 1 these predicates can be combined with "or" and "and" by using a logical predicate operator. Other tuple-based operators include the derive $(f)$ and the unnest $(\mu)$ operator. The derive operator applies a function to a single tuple and appends the result value to the tuple. In our case, we use the derivation functions to add the terms included in the content attribute of tuple and the sentiment of the content attribute of the tuple to the tuple. The unnest operator splits a "nested" attribute value and emits a tuple for each new value. A typical use case for the unnest operator is to split a string and to produce a tuple for each term it contains. Apart from these general operators, Niagarino provides a number of stream-specific operators that can be used to segment the unbounded stream for processing. Apart from the well-known time and tuple-based window operators $(\omega)$ that can be tumbling or sliding ( $\mathrm{Li}$ et al., 2005), Niagarino also implements data-driven windows, so-called frames (Maier et al., 2012). For the Twitter case study (see Section 4.1), we use timebased and for the Harry Potter case study (see Section 4.2) chapter-based tumbling windows.

Stream segments form the input for join $(\bowtie)$ and aggregation $(\Sigma)$ operators. As with derive operators, Niagarino also supports user-defined aggregation functions. Niagarino operators can be partitioned into 


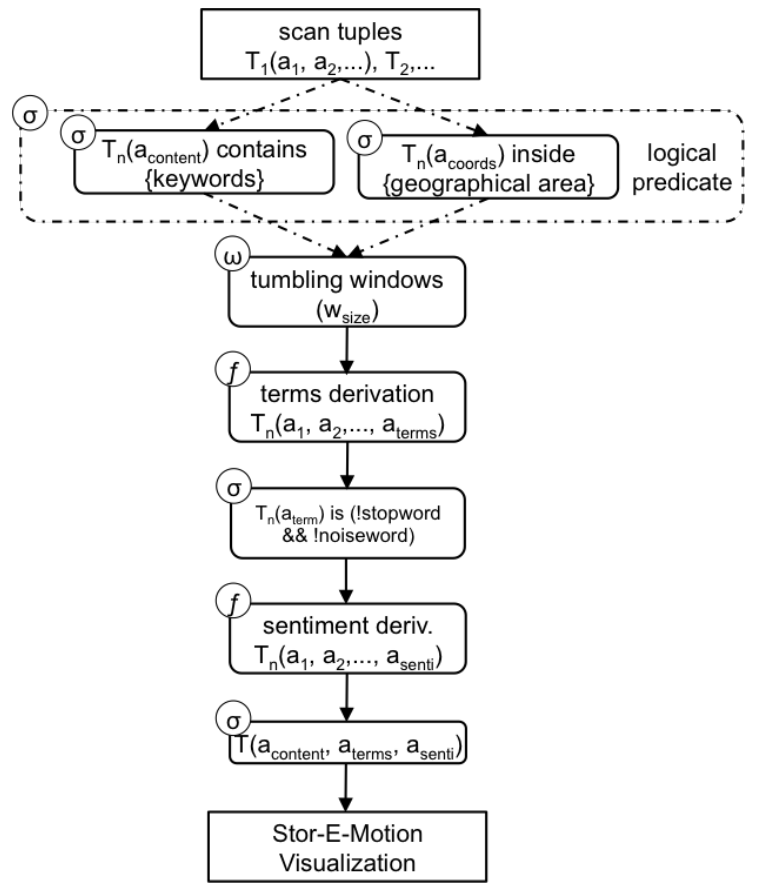

Figure 1: Query plan of a single topic channel.

three groups. The operators described above are general operators, whereas source operators read input streams and sink operators output results. Each query can have multiple source and sink operators. Source and sink operators used in the processing pipeline are shown as rectangle in Figure 1.

\subsection{VISUALIZATION}

To visualize the evolution of topics over time and to retain the original sequence of the text stream, we use a shape based visualization in which all three major design goals are incorporated. The visualization is tailored to point the user to important and interesting patterns in the streaming data and also to support the user in serendipitous findings in the topics. The major design goals are described in the following.

- Real-time visualization of the topic's evolution of frequency and emotion

- Presentation of the topic's story in a compact but significant way

- Detail view of the story's content

Figure 2 shows the shape, which consists of a rounded rectangle, which reflects the frequency and the percentages of the sentiment values of the time window. These shapes are continuously added to the next position at the right side in the panel and therefore form different patterns by visual aggregation in the ongoing time series of the topic.

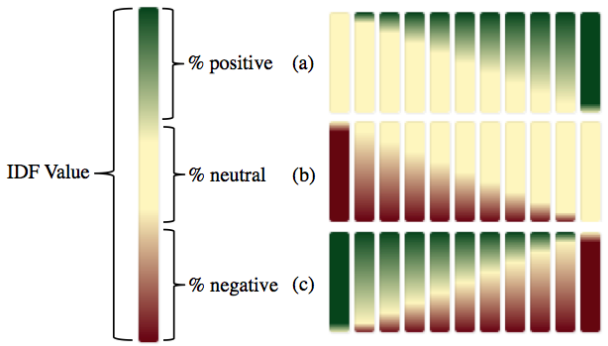

Figure 2: Left: Shape for a single data window with $40 \%$ positive and $40 \%$ negative sentiment; Right: Examples for (a) increasing \% positive, (b) decreasing \% negative, (c) decreasing $\%$ positive and increasing $\%$ negative sentiment.

The examples in Figure 2 show a time series with unchanged frequency but with increasing positive sentiment (a), decreasing negative sentiment (b), and decreasing positive and increasing negative sentiment (c).

\section{IMPORTANCE}

The visualization of the ongoing topic evolution needs to reflect the continuous and dynamic change of the importance of a topic over time. Therefore, the importance of the topic in the time window is visualized by using the size of the shape. Since the length of a time window is pre-defined and static, we use this value as the width of the rectangle. For the height of the rectangle, we calculate for each shape a value against a pre-defined static max_height. To calculate this value, we use the values $n$ (total number of tuples inside the window) and $m$ (total number of tuples inside the topic channel and window) and calculate the Inverse Document Frequency (Sparck Jones, 1988) for both values. Then we use the two frequency values in the following formula to get the height value.

$$
\text { height }=\left(\log \left(\frac{n}{1}\right)-\log \left(\frac{n}{m}\right)\right) *\left(\frac{\text { max_height }}{\log \left(\frac{n}{1}\right)}\right)
$$

\section{EMOTION}

The emotion of a topic is visualized by using the coloring of the shapes. The filling color of the shape signifies the average sentiment (red $=$ negative, green $=$ positive, yellow $=$ neutral $($ see Figure 2$)$ of the text in the data window. The value of the sentiment for a text segment is calculated by using an external library et al. (Thelwall et al., 2010). The library analyzes the text of the message and returns values for the sentiment between -4 to -1 (extremely negative to negative), $O$ (neutral), and 1 to 4 (positive to extremely 
positive). To reflect the different levels of the sentiment, we calculate a stepwise $(0.1$ steps $)$ color value corresponding to the average value of all positive as well as all negative values. The darker the color the higher is the average value. The color mapping can be seen at the left side of each topic channel.

To visualize the ratio of positive and negative sentiment in the data windows, we use a linear gradient with the colors according to the color map and the percentage of positive or negative text segments in the overall text in the window. For example, Figure 2 shows a shape for a single window, which has $40 \%$ of extremely negative and $40 \%$ of extremely positive sentiment.

\section{STORY}

Since the story around a topic also evolves over time, it is a challenging task to keep an overview of ongoing context changes in the topic channels. Therefore, we visualize the story by using a tag list for a predefined number of data windows in a continuous way. We call this collection of windows an episode and the included content episode terms.

The tag list is created by tokenizing the contents of the text segments and removing terms which are included in a standard English stopword list or are classified as noise terms (terms that are too short or too long, terms with a repetition of the same character, or terms without a vowel). Furthermore, we filter out terms that are included in the topic channel definition, because these terms would always be very frequent in the resulting term set. To reflect the ongoing evolution of terms around a topic, we use two rules to increase the importance of newly occurring or in their frequency increasing terms. The newly occurring terms are multiplied with the average frequency value of all terms from the previous window and the already seen terms (in previous windows) are multiplied by their increasing factor. By taking the top five terms of our computation to be shown for the corresponding time window, we ensure that only terms are displayed that have a certain influence to the story of the topic.

The resulting episode terms are added to the space of the corresponding episode. Since single terms, even if they are in a group of episode terms, are sometimes not self-explanatory and it is therefore very helpful to obtain additional context information of single terms, we added an overview of the respective texts for selected terms. For example, in the city observation case study (see Section 4.1) of Boston, we are able to get an insight into the first on-site reports about the explosion (including a hyperlink to the very first image about the event). Additionally, each single text in the overview is colored with it corresponding sentiment color. This approach supports the user in getting a better insight, into how much each text content has contributed to the overall sentiment value.

\subsection{TOPIC CHANNELS}

A topic can be defined in several ways. In text streams, in which the only source is the text, the topic definition mostly consists of one or several keywords. However, by using social media data, which contain a large amount of additional meta-data, topics can be defined in a great variety. For example, a topic channel could be defined by the rules to follow specific Twitter users in terms of their geographic location or timezone. However, since we are interested in the evolution of important and specific topics, we focus on the textual and the geographical dimension of the data stream. The three possible topic channel definitions are described in the following.

Keywords Hereby, we can create a topic channel, which follows a topic about one or several keywords. There exist two alternatives for this channel definition. First, the text segment needs to contain all of the defined keywords ("and" clause) or second it needs to contain at least one ("or" clause) of the keywords.

Geographic We can also create a topic channel, which follows topics in one or several geographical areas. For example, it is possible to define a geographical location as the center and radius of the surrounding area to observe the importance, emotions, and story inside a certain city or country. Note, that in this case only the "or" clause is reasonable.

Mixed To observe topics in a certain geographically area more precisely, it is also possible to combine the textual and the geographical filtering. For example, if a user is interested in an earthquake in a certain country and not all over the world, it is for example possible to query for the keyword "earthquake" and a country definition of Indonesia.

Since the ongoing evolution of the topic's story, which eventually deals with important subtopics and surfaces serendipitous findings, can trigger the interest in new topics, it is always possible to add new topic channels with a totally new definition or extend/restrict topic definitions of existing topics. By using the Niagarino framework we can easily change or substitute the definitions of channels and also combine different types of channel definitions by using a logical predicate operator. 


\section{CASE STUDIES}

In this section, we present case studies for two different data sets. In the first three case studies, we use the public live text data stream of Twitter. The fourth and final case study uses the entire text of all volumes of the fantasy book series "Harry Potter" as a text data stream.

\subsection{TWITTER STREAM}

The Twitter platform provides direct access to the public live stream of Twitter. By using the Twitter Streaming API ${ }^{2}$ with the so-called "Gardenhose" access level, we receive a randomly sampled $10 \%$ stream of the public live stream. An exemplary evaluation of a representative sample of days shows that the $10 \%$ stream contains an average of over 1.5 million tweets per hour with an average of 25,000 tweets per minute. We can also conclude that there is an increasing availability of tweets with geographic information with currently about 2,000 to 4,000 of incoming tweets per minute. The geographic information either consists of the latitude and longitude value, which is automatically set by the used mobile device or a location manually added to the tweet by the author of the message. The live creation of a single topic channel for a 24 hour period of data takes about 15 minutes $(3,2 \mathrm{GHz}$ Intel Core i3 processor and $8 \mathrm{~GB}$ of main memory) to process all tuples. The parallel creation of the four topic channels of the first case study took about 23 minutes. Therefore, we can conclude that, while it is still possible to follow the live stream of tweets, adding further topic channels slows down the processing.

For the following case studies, we collected the tweets as they are streamed out by the API for the specific dates in the central Europe time zone (CET). Because the sentiment derivation function only works for English texts, we pre-filtered the data sets for tweets whose content is in English by using a language detection library ${ }^{3}$. After the pre-processing, we get a tuple stream $\left\{T_{1}, \ldots, T_{n}\right\}$ in which each tuple has the attributes $\left\{a_{i d}, a_{\text {creationdate }}, a_{\text {content }}, a_{\text {coords }}\right\}$. Note that the coordinates attribute $\left(a_{\text {coords }}\right)$ is only set if the tweet contains geographic information. The visualizations are created in the following manner. For each tuple flowing through our processing pipeline the topic definitions are checked and terms as well as the sentiment value is derived from the $a_{\text {content }}$ attribute. After this pre-processing each tuple has the attributes

\footnotetext{
2 https://dev.twitter.com/streaming/overview

3 http://code.google.com/p/ language-detection/
}

$\left\{a_{\text {id }}, a_{\text {creationdate }}, a_{\text {content }}, a_{\text {coords }}, a_{\text {terms }}, a_{\text {sentiment }}\right\}$.

The $w_{\text {size }}$ value of the tumbling window operator is set to one minute and therefore a shape reflects the aggregated values of one minute consisting of the importance and emotion for the topics. The width of the shape is set to two pixels and the max_height to 120 pixels. For each episode of 60 minutes a tag list with the most influencing episode terms is displayed. By selecting an episode term, the content and sentiment of all tweets that belong to this term and episode are shown in the detail view (see Figure 4 for example).

\section{CITY OBSERVATION}

The first case study describes the observation of the city Boston on the day April $15^{\text {th }}, 2013$. The data set for that day contains a total of 20,046,861 tuples, which is an average of about 830,000 tuples per hour and about 14,000 tuples per minute. Figure 3 shows the visualization of four topics for the whole day. The first topic channel Boston (Geo) is defined by using the city center of Boston and the surrounding area of 25 miles. The second topic channel Boston is defined by using the name of the city as keyword. These are the two starting topic channels a news reporter is likely to choose in order to follow the events around a specific city. These definitions make it possible to get an overview of the tweets using the name of the city Boston as well as the tweets that are sent from within the city (and probably report on-site reports), but that do not include the name of the city in the content of the message.

By observing the ongoing evolution of the topic channel Boston (Geo) and Boston, we can see that the frequency decreases after a couple of hours. By that time it was night in Boston and people sending tweets from Boston or about Boston are less active. The story after these low frequency episodes in both channels mostly consists of episode terms related to a sports event (e.g., "running", "team", and "marathon"). Attracted by the term "marathon", we are also interested in following this topic. Therefore we choose to follow this term (see the blue rectangle in Figure 3 inside the Boston (Geo) topic channel) and a new topic channel with the title Marathon appears. In this topic channel, we can now see more details about the marathon event. In the Marathon topic channel, we can identify the episode term "desisa" and "ethiopia". Also in the Boston (Geo) the episode term "jeptoo" is mentioned. By getting more insights into these terms by using the detail view of the tweets, we can derive that these are the winners of the marathon. The most interesting pattern appears a few hours after these first runners finished the marathon. 


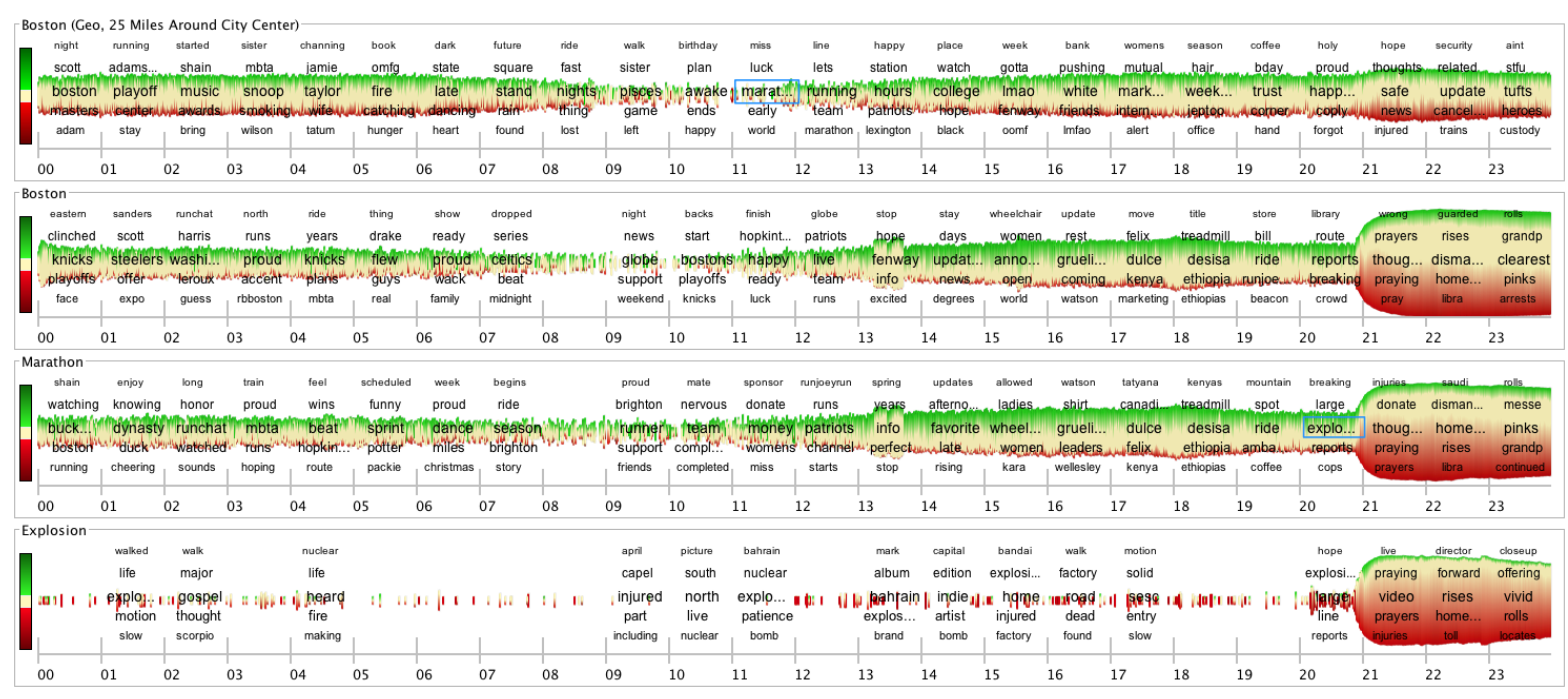

Figure 3: Visualization of the topic channels (Twitter stream from April 15 $5^{\text {th }} 2013$ ) of Boston (Geo), Boston, Marathon, and Explosion from top to bottom. Blue rectangles are the activators to start the corresponding topic channels.

The negative emotion of all three topic channels increases and drifts into the extremely negative. Also, the overall importance of all three topic channels increases significantly and therefore reflects the happening of an interesting event. In the Marathon topic channel, we are now attracted by the term "explosion" and again add a new topic channel for this term. We can derive that, at this point in time, an explosion took place at the finishing line (episode term "line" appears in the tag list) of the marathon course.

Shortly afterwards the terms "prayers", "cops", and "injured" appear in the tag list of the different channels. These terms reflect the current situation in the ongoing topic. By having a look at the episode terms of the Boston (Geo) we can see that the term "coply" (Copley square is a public place in the city of Boston) appears in the episode before the event. The detail view of this term (see Figure 4) shows the first two on-site reports, which are written very shortly after the event, about the event (with hyperlinks to the first on-site pictures). It also indicates that the earlier tweets about "coply" are positive or neutral and the latter are changed to negative. We can derive that our series of topic channels effectively reflects the parallel evolution of the ongoing events in the city Boston.

\section{ELECTION OBSERVATION}

The second case study describes the observation of the papal election on the day March $13^{\text {th }}, 2013$. The dataset for that day contains a total of 19,470,337 tuples, which is an average of about 810,000 tuples per hour and about 13,500 tuples per minute. Figure 5 shows the visualization of two topics for the whole

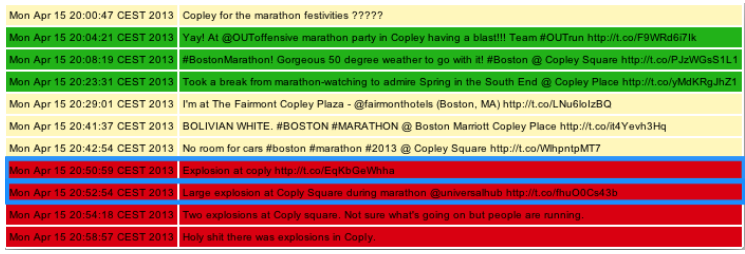

Figure 4: Detail view of the episode term "coply" from the topic channel Boston (Geo) in the episode 21.

day. The source topic channel Pope is defined by the rule that a tweet needs to contain at least one of the keywords "pope" or "pontifex".

By observing the ongoing evolution of the topic channel Pope, we can clearly see that the frequency stays almost constant until the point of time when the term "whitesmoke" (term is used as hashtag by the Twitter community to signal that the papal election is finished and a new pope is announced to the world) appears in the tag list in Episode 19 and the frequency increases significantly. The co-occurring episode terms "habemus" and "papam" (latin phrase for a successful pope election) imply the same.

In the next episode, the tag list of the topic channel shows the term "argentina" and therefore we are also interested in the topic evolution of this topic. We can recognize in the topic channel Argentina that the election meets the people in Argentina with a positive response. Furthermore, we can see that the burst of tweets about the election event takes place in Argentina almost one hour later than the event is reported in the source topic channel. Also two more indicators for that event like "pope" and "elected" appear in Episode 20 in the Argentina topic channel. 


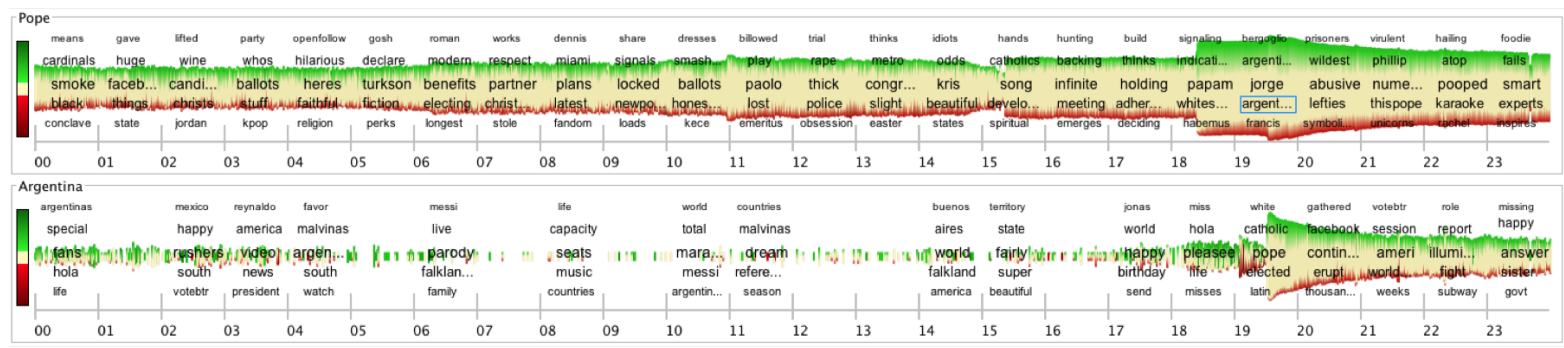

Figure 5: Visualization of the topic channels (Twitter stream from March $13^{\text {th }}$ 2013) Pope and Argentina.

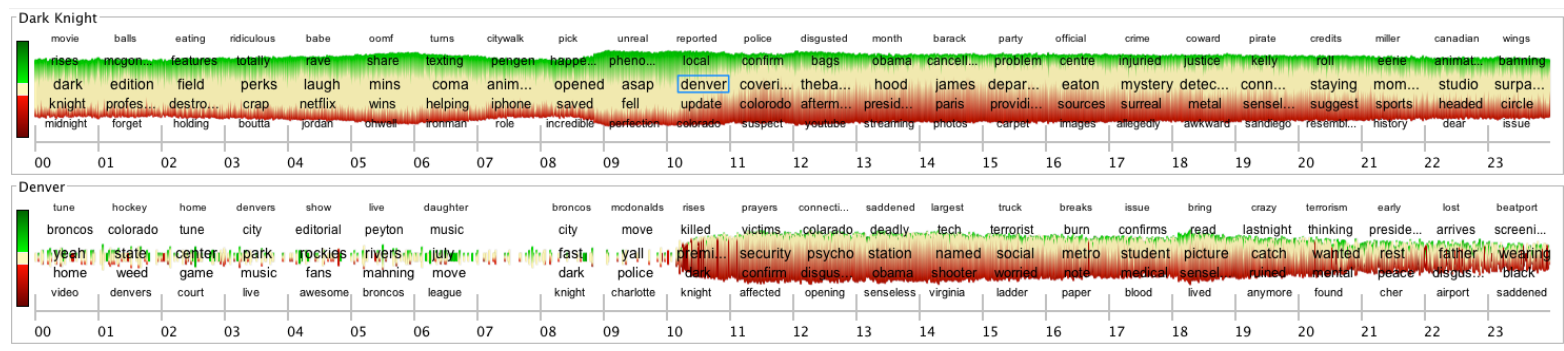

Figure 6: Visualization of the topic channels (Twitter stream from July $20^{\text {th }} 2012$ ) Dark Knight and Denver.

\section{MOVIE PREMIERE OBSERVATION}

The third use case describes the observation of a movie premiere on the day July $20^{\text {th }}, 2012$. The dataset for that day contains a total of $12,760,600$ tuples, which is an average of about 530,000 tuples per hour and about 9,000 tuples per minute. Figure 6 shows the visualization of three topics for the whole day. The source topic channel Dark Knight is defined by using the keywords "dark knight" and "batman"

The evolution of the topic channel Dark Knight reflects the ongoing movie premiere of the movie "The Dark Knight Rises", which premiered on that day. We can identify that there is a slight increase (in Episode 10) in the frequency within the time frame when the premiere starts in the cinema. The emotion per minute in the time frame at the beginning tends to be more positive than negative because of the anticipation of the movie. However, after a short period of time (starting with Episode 11) the sentiment drifts to the negative and the episode term "denver" appears in the tag list. This triggers us to start the new topic channel Denver. The unexpected increase in negative sentiment is a clear sign that something unexpected happened.

In the Denver channel episode terms like "killed", "victims", and "security" appear in the Episodes 11 and 12. An interesting observation is that the frequency and the negative sentiment increases significantly for the Denver topic channel, while for the Dark Knight topic channel only the negative sentiment increases. Also we note the term "james" that appears in Episode 15 of the first topic channel, which we are able to determine (by inspecting the detail view of the tweets) as the name of the suspect.

\subsection{TEXT STREAM}

Another promising application for a streaming text visualization are single books or complete book series. Therefore, we use this kind of data to further evaluate our visualization and perform a final case study, which uses the complete text of the fantasy book series of "Harry Potter" as text data stream. We pre-processed the complete book series and extracted a total of 195 book chapters with a total of 33,939 sentences that contain more than ten characters. After the pre-processing step we get a tuple stream $\left\{T_{1}, \ldots, T_{n}\right\}$ in which each tuple has the attributes $\left\{a_{\text {chapter }}, a_{\text {book }}, a_{\text {content }}\right\}$. For each tuple, the topic definitions are checked and the terms as well as the sentiment value is derived. The derivation processes add the new attributes $a_{\text {terms }}$ and $a_{\text {senti }}$ to each tuple. The $w_{\text {size }}$ value of the tumbling window operator is set to one in order that a shape reflects the aggregated sentiment values of one chapter, consisting of the importance and emotion for the topics. For each episode of five chapters a tag list with the most influencing episode terms is displayed. By selecting an episode term, all sentences that belong to that term and episode are shown and are colored in the corresponding sentiment value. The creation of the entire visualization takes about 30 seconds. Figure 7 shows the visualization for all topic channels that we used in our exploration. Note, that for this case study there is more horizontal space available per data window 
and therefore we set the width of a shape to 12 pixel (max_height is still 120 pixels).

The first topic channel shows the overall evolution of the importance, emotion, and story of the book series. Since the height of the shape is normalized to the total amount of sentences in the chapter, there is no change in the shapes height for this channel. However, the overall channel supports users in reviewing the full evolution of emotion and story as well as in finding potential terms, which we might be interested in following and exploring in further topic channels.

By tracking the episode terms of the overall channel, we see that the term "harry" is influential in the first episode and add this term as a second topic channel to the visualization. In this channel, we directly recognize two additional terms in the first two episodes. First, the term "hagrid" and second the term "hermione" are used to build new topic channels. The topic channels Hermione and Harry clearly reflect the interplay between these characters in the story. By further tracking the episode terms of the Overall channel, we can identify the term "kill" in the Episode 11. In order to explore the term "kill", we have a look at the corresponding sentences and can recognize that the "death" of characters is an always important topic in the story. Therefore, we add another topic channel, which we call Kill and that is filtered by the keywords "kill, death, died, killed, or dead". Since these are all terms which are related to negative things the topic channel for this topic is mainly colored in red. However, we can still recognize the uneven appearance of the topic in the ongoing book series and detect some extremely negative patterns. The next topic channel (Dobby) is also added as a consequence of the occurrence of this term in the Overall channel.

By following the Dobby channel, we see the episode term "hogwarts" as one of the influencing terms in the story of this channel. As we assume this to be an interesting term, we also track this term within a new topic channel. In this channel, we can see the episode term "horcrux" in the second-last episode, which also seems to be important and therefore we add this term as the last topic channel to the visualization. This leads to the observation that there are very unevenly distributed topics in the full story. The topic channel eight directly reveals that Horcrux (magical objects) have only been introduced in the last books of the fantasy series. Also, we can derive that the character dobby only occurs occasionally and leaves the story with a shape in negative color. By looking at the corresponding sentences, we can derive that these shapes signify the death of the character.

\section{CONCLUSION}

In this paper, we presented "Stor-e-Motion", a shape-based visualization to track the ongoing evolution of topics' frequency (i.e., importance), sentiment (i.e., emotion), and context (i.e., story) in userdefined topic channels over continuously flowing text data streams. Our case studies show that the visualization supports users in keeping the overview in following and tracking topics over time and also guides them to interesting points or periods in time. Furthermore the visualization contributes to a common, timely, and relevant situational awareness of topics and allows them for serendipitous findings. These findings can be easily added to existing or form new topic channels and therefore support the refinement of topics.

Future work includes an evaluation and a user study of the visualization and to further extend the analytical functionality. A possible idea would be to additionally include an event detection mechanism and automatically feed the resulting terms into the visualization to create a large landscape of events and topics.

A further improvement would be to extend the system with a zooming feature to provide a more detailed view to the users. This allows data to be displayed at different levels of granularity in order to get deeper insights into the interesting points or periods in time. For the topic channel definition, further options, such as the source of the tweet (e.g., mobile phone or web), the geographic region of the tweet or the type of the tweet (e.g., retweet or direct message), could be derived from the meta-data of tweets.

For a more powerful search and to improve the results, more full-text options, such as fuzzy search or the exclusion of negative terms could be added. The importance of the exclusion of negative terms can be derived from the city observation case study, in which the results shifted from tweets about the original topic (reactions to the sport event Marathon) to a different topic (reactions to the explosions) and therefore it would be helpful to separate the topics from each other.

\section{REFERENCES}

Abadi, D. J., Ahmad, Y., Balazinska, M., Çetintemel, U., Cherniack, M., Hwang, J., Lindner, W., Maskey, A., Rasin, A., Ryvkina, E., Tatbul, N., Xing, Y., and Zdonik, S. B. (2005). The Design of the Borealis Stream Processing Engine. 


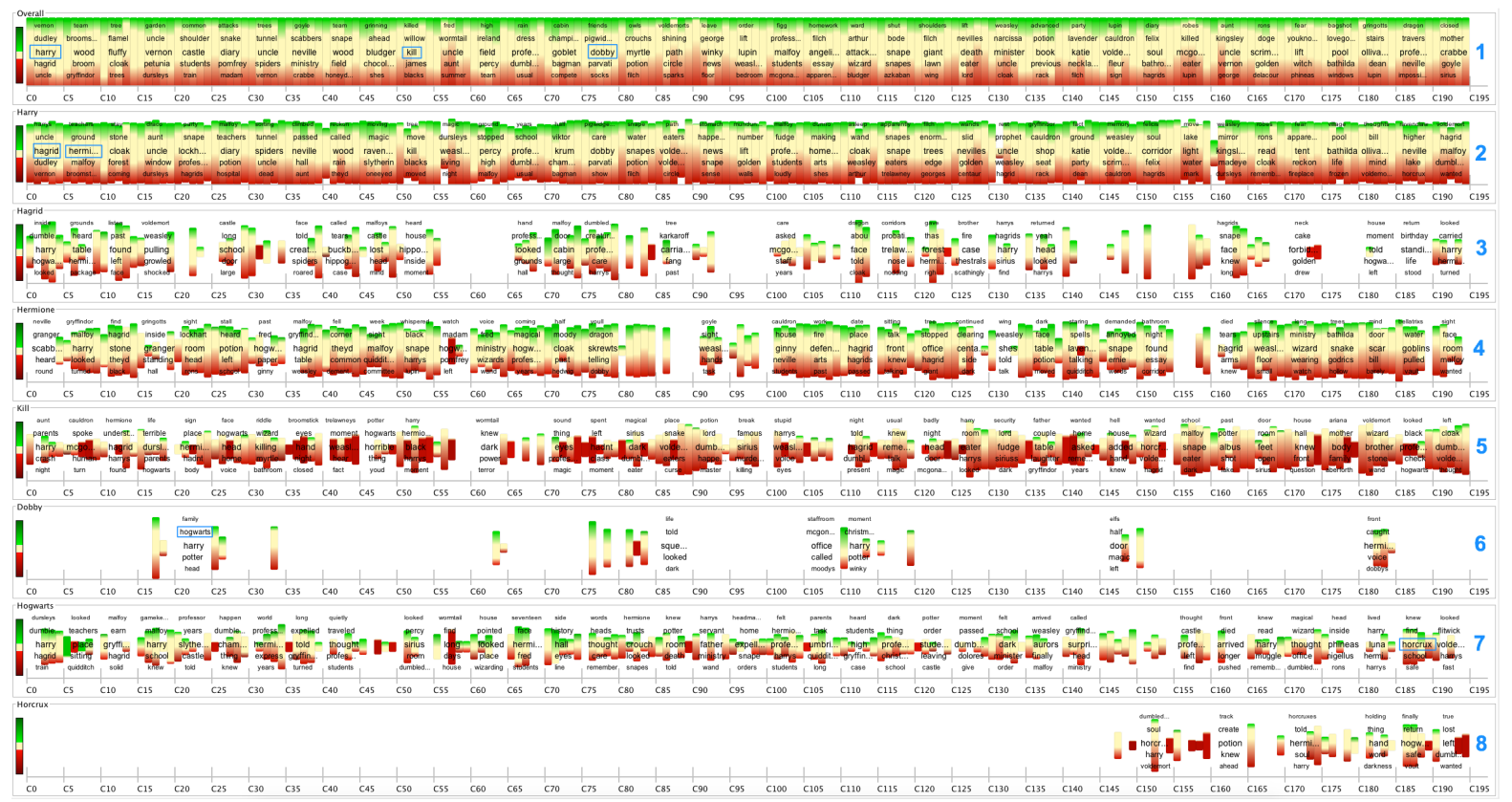

Figure 7: Visualization of the text data stream from the complete fantasy book series Harry Potter. It shows the overall evolution of the story without any defined topic (first line) and the evolution in the topic channels of Harry, Hagrid, Hermione, Kill, Dobby, Hogwarts, and Horcrux from top to bottom. Blue rectangles are the activators to start the topic channels.

In Proc. Intl. Conf. on Innovative Data Systems Research (CIDR), pages 277-289.

Abadi, D. J., Carney, D., Çetintemel, U., Cherniack, M., Convey, C., Lee, S., Stonebraker, M., Tatbul, N., and Zdonik, S. (2003). Aurora: A New Model and Architecture for Data Stream Management. The VLDB Journal, 12(2):120-139.

Alvanaki, F., Michel, S., Ramamritham, K., and Weikum, G. (2012). See what's enblogue: realtime emergent topic identification in social media. In Proceedings of the 15th International Conference on Extending Database Technology, EDBT '12, pages 336-347, New York, NY, USA. ACM.

Arasu, A., Babu, S., and Widom, J. (2006). The CQL Continuous Query Language: Semantic Foundations and Query Execution. The VLDB Journal, 15(2):121-142.

Bontcheva, K. and Rout, D. (2012). Making sense of social media streams through semantics: a survey. In Semantic Web journal.

Bosch, H., Thom, D., Heimerl, F., Puttmann, E., Koch, S., Krüger, R., Wörner, M., and Ertl, T. (2013). Scatterblogs2: Real-time monitoring of microblog messages through user-guided filtering. IEEE Trans. Vis. Comput. Graph., 19(12):2022-2031.

Culotta, A. (2010). Towards detecting influenza epidemics by analyzing twitter messages. In Pro- ceedings of the First Workshop on Social Media Analytics, SOMA '10, pages 115-122, New York, NY, USA. ACM.

Dork, M., Gruen, D., Williamson, C., and Carpendale, S. (2010). A visual backchannel for largescale events. IEEE Transactions on Visualization and Computer Graphics, 16(6):1129-1138.

Havre, S., Hetzler, E., Whitney, P., and Nowell, L. (2002). Themeriver: Visualizing thematic changes in large document collections. Visualization and Computer Graphics, IEEE Transactions on, 8(1):9-20.

Java, A., Song, X., Finin, T., and Tseng, B. (2007). Why we twitter: understanding microblogging usage and communities. In Proceedings of the 9th WebKDD and 1st SNA-KDD 2007 workshop on Web mining and social network analysis, pages 56-65. ACM.

Krstajić, M., Bertini, E., and Keim, D. A. (2011). Cloudlines: Compact display of event episodes in multiple time-series. IEEE Trans. Vis. Comput. Graph., 17(12):2432-2439.

Kwak, H., Lee, C., Park, H., and Moon, S. (2010). What is Twitter, a social network or a news media? In Proceedings of the 19th international conference on World wide web, WWW '10, pages 591-600. ACM.

Lee, S., Lee, S., Kim, K., and Park, J. (2012). Bursty 
event detection from text streams for disaster management. In Proceedings of the 21st international conference companion on World Wide Web, WWW '12 Companion, pages 679-682, New York, NY, USA. ACM.

Li, J., Maier, D., Tufte, K., Papadimos, V., and Tucker, P. A. (2005). No Pane, No Gain: Efficient Evaluation of Sliding-Window Aggregates over Data Streams. SIGMOD Record, 34(1):3944.

Li, J., Tufte, K., Shkapenyuk, V., Papadimos, V., Johnson, T., and Maier, D. (2008). Outof-Order Processing: A New Architecture for High-Performance Stream Systems. PVLDB, 1(1):274-288.

MacEachren, A., Jaiswal, A., Robinson, A., Pezanowski, S., Savelyev, A., Mitra, P., Zhang, X., and Blanford, J. (2011). Senseplace2: Geotwitter analytics support for situational awareness. In Visual Analytics Science and Technology(VAST), 2011 IEEE Conference on, pages 181-190.

Maier, D., Grossniklaus, M., Moorthy, S., and Tufte, K. (2012). Capturing Episodes: May the Frame Be With You. In Proc. Intl. Conf. on Distributed Event-Based Systems (DEBS), pages 1-11.

Marcus, A., Bernstein, M. S., Badar, O., Karger, D. R., Madden, S., and Miller, R. C. (2011). Twitinfo: aggregating and visualizing microblogs for event exploration. In Proceedings of the 2011 annual conference on Human factors in computing systems, CHI '11, pages 227-236. ACM.

Naaman, M., Boase, J., and Lai, C.-H. (2010). Is it really about me?: message content in social awareness streams. In Proceedings of the 2010 ACM conference on Computer supported cooperative work, CSCW'10, pages 189-192. ACM.

Overby, D., Keyser, J., and Wall, J. (2009). Interactive visual analysis of location reporting patterns. In Visual Analytics Science and Technology, 2009.
VAST 2009. IEEE Symposium on, pages 223 224. IEEE.

Ritter, A., Mausam, Etzioni, O., and Clark, S. (2012). Open domain event extraction from twitter. In Proceedings of the 18th ACM SIGKDD international conference on Knowledge discovery and data mining, KDD '12, pages 1104-1112, New York, NY, USA. ACM.

Sakaki, T., Okazaki, M., and Matsuo, Y. (2010). Earthquake shakes Twitter users: real-time event detection by social sensors. In Proceedings of the 19th international conference on World wide $w e b$, WWW'10, pages 851-860. ACM.

Sparck Jones, K. (1988). A statistical interpretation of term specificity and its application in retrieval, pages 132-142. Taylor Graham Publishing.

Thelwall, M., Buckley, K., Paltoglou, G., Cai, D., and Kappas, A. (2010). Sentiment strength detection in short informal text. Journal of the American Society for Information Science and Technology, 61(12).

Tonkin, E., Pfeiffer, H. D., and Tourte, G. (2012). Twitter, information sharing and the London riots? Bulletin of the American Society for Information Science and Technology, 38(2):49-57.

Wanner, F., Ramm, T., and Keim, D. A. (2011). Foravis: Explorative user forum analysis. In Proceedings of the International Conference on Web Intelligence, Mining and Semantics, WIMS '11, pages 14:1-14:10, New York, NY, USA. ACM.

Wanner, F., Rohrdantz, C., Mansmann, F., Stoffel, A., Oelke, D., Krstajic, M., Keim, D. A., Luo, D., Yang, J., and Atkinson, M. (2009). Largescale Comparative Sentiment Analysis of News Articles (InfoVis 2009). Poster at IEEE InfoVis 2009.

Weng, J., Yao, Y., Leonardi, E., and Lee, F. (2011). Event Detection in Twitter. Technical report, HP Labs. 